\title{
Killing the cMSSM softly
}

\section{Michael Krämer}

Institut für theoretische Teilchenphysik und Kosmologie, RWTH Aachen, Germany

E-mail: mkraemerephysik.rwth-aachen.de

Philip Bechtle, Klaus Desch, Herbi Dreiner, Björn Sarrazin, Matthias Uhlenbrock, Peter Wienemann

Physikalisches Institut, Universität Bonn, Germany

E-mail: bechtledphysik.uni-bonn.de, deschlphysik.uni-bonn.de, dreinereth.physik.uni-bonn.de, sarrazin@physik.uni-bonn.de, uhlenbrock@physik.uni-bonn.de, wienemann@physik.uni-bonn.de

Matthias Hamer*

Brazilian Center for Physics Research, Rio de Janeiro, Brazil

E-mail: matthias.hamerdcern.ch

\section{Tim Stefaniak}

Santa Cruz Institute for Particle Physics, California, USA

E-mail: tistefan@ucsc.edu

\section{Jose Camargo-Molina, Ben O’Leary, Werner Porod}

Institut fur Theoretische Physik und Astrophysik, University of Würzburg, Germany

E-mail: jose.camargo@physik.uni-wuerzburg.de,

ben.olearyaphysik.uni-wuerzburg.de,

porodephysik.uni-wuerzburg.de

The analysis of the data collected by the major LHC experiments during the LHC Run I has put strong constraints on supersymmetric models. We study the parameter space of the constrained Minimal Supersymmetric Standard Model (cMSSM) in a global fit, taking into account the nonobservation of supersymmetry at the LHC, Higgs mass and rate measurements, as well as several cosmological and low energy observables. Before the start of the LHC, global fits of the cMSSM showed a favourable goodness-of-fit and indicated a strong preference for the existence of light SUSY particles. This region now has largely been excluded by the LHC. We present the final results of our study of the status of the cMSSM after the LHC Run1, where for the first time we use pseudo experiments to determine the p-value of the model. We find that the cMSSM is softly getting near its exclusion at the $90 \%$ CL.

The European Physical Society Conference on High Energy Physics

22-29 July 2015

Vienna, Austria

\footnotetext{
*Speaker.
} 


\section{Introduction}

Amongst the multitude of models that exist for supersymmetric [1] extensions of the Standard Model (SM), the cMSSM [2] is one of the most simple and best studied models. Defined at the GUT scale, its parameter space consists of a common mass $M_{0}$ for scalars, a common mass $M_{1 / 2}$ for fermions, a common proportionality factor $A_{0}$ for trilinear couplings and the ratio of the vacuum expectation values of the two Higgs doublets $\tan \beta$. In addition there is one discrete parameter, sign $\mu$.

Supersymmetric models can be tested with a number of measurements from different areas; apart from direct searches at collider experiments, low energy and precision observables, the properties of the discovered Higgs boson as well as measurements from astrophysics can be used to constrain the parameter space.

The non-observation of any superpartners during the LHC Run1 has introduced some tension between these areas within the cMSSM [3]. Leading to an increase of the minimal $\chi^{2} /$ ndof, this prompts the question if the cMSSM can be excluded. As it is not obvious that the minimal $\chi^{2}$ is distributed according to a Gaussian $\chi^{2}$-distribution with ndof degrees of freedom, we use pseudo experiments to determine the $\mathrm{p}$-value.

This article is organised as follows: In section 2, the used observable set is summarised. In section 3 the techniques for scanning the parameter space and determining the $p$-value are described. An overview of the fit results and the obtained $\chi^{2}$-distribution and p-value are presented in section 4 .

\section{Observable Sets and Predictions}

Table 1 shows the low energy observables used in the fit. We assume that the lightest supersymmetric particle (LSP) accounts for the full amount of dark matter and thus reject points where the $\tilde{\chi}_{1}^{0}$ is not the LSP. In addition to the dark matter relic density the limit on the spin-independent nucleon-dark matter scattering cross-section set by the LUX experiment is taken into account [4]. We also consider a lower bound on the mass of the $\tilde{\chi}_{1}^{+}$of $103.5 \mathrm{GeV}$ as set by the LEP experiments [5], as well as the direct search for SUSY in final states with zero leptons, jets and missing transverse energy by the ATLAS experiment [6]. Limits on the Higgs masses are taken into account via the program HigGSBounds [7]. Finally we consider available measurements of the signal strengths and the mass of the Higgs boson found by the ATLAS and CMS experiments as implemented in the program HigGSSigNALS [8]. Here we use combined measurements in the decay channels $\mathrm{H} \rightarrow \gamma \gamma, \mathrm{H} \rightarrow \mathrm{ZZ}, \mathrm{H} \rightarrow \mathrm{WW}, \mathrm{H} \rightarrow \tau \tau$ and $\mathrm{VH} \rightarrow \mathrm{Vbb}$ from ATLAS and CMS separately. For the calculation of the sparticle mass spectrum we use SPHENO 3.2.4 [9]. Higgs boson masses and properties are calculated with FEYNHiggs 2.10.1 [10]. The B-physics observables are calculated with SUPERISO 3.3 [11]. We use MicrOMEGAs 3.6.9 [12] for the calculation of the dark matter relic density and DARKSUSY 5.0.5 [13] via ASTROFIT [14] for the direct detection cross section. The ATLAS analysis is emulated using HERWIG++ [15] to generate the events, DELPHES [16] for a fast detector simulation and PROSPINO [17] for the calculation of the gluino and squark production cross sections. 


\begin{tabular}{lllc} 
Observable & Measurement & Uncertainty & \\
\hline$a_{\mu}-a_{\mu}^{\mathrm{SM}}$ & $(28.7 \pm 8.0) \times 10^{-10}$ & $7 \%$ & {$[18,19]$} \\
$\sin ^{2} \theta_{\text {eff }}$ & $0.23113 \pm 0.00021$ & $0.05 \%$ & {$[20]$} \\
$m_{t}$ & $(173.34 \pm 0.27 \pm 0.71) \mathrm{GeV}$ & $1 \mathrm{GeV}$ & {$[21]$} \\
$m_{W}$ & $(80.385 \pm 0.015) \mathrm{GeV}$ & $0.01 \%$ & {$[22]$} \\
$\Delta m_{s}$ & $(17.719 \pm 0.036 \pm 0.023) \mathrm{ps}^{-1}$ & $24 \%$ & {$[23]$} \\
$\mathscr{B}\left(B_{s} \rightarrow \mu \mu\right)$ & $(2.90 \pm 0.70) \times 10^{-9}$ & $26 \%$ & {$[24]$} \\
$\mathscr{B}(b \rightarrow s \gamma)$ & $(3.43 \pm 0.21 \pm 0.07) \times 10^{-4}$ & $14 \%$ & {$[25]$} \\
$\mathscr{B}(B \rightarrow \tau v)$ & $(1.05 \pm 0.25) \times 10^{-4}$ & $20 \%$ & {$[23]$} \\
$\Omega h^{2}$ & $0.1187 \pm 0.0017$ & $10 \%$ & {$[26]$} \\
\hline
\end{tabular}

Table 1: Precision observables used in the fit.

\section{Scan of the Parameter Space}

The parameter space of the cMSSM is scanned by a Markov Chain Monte Carlo (MCMC) with Gaussian proposal density functions with a varying width. Measurements and predictions are compared at each point in the MCMC by means of the total $\chi^{2}$,

$$
\chi^{2}=\left(\vec{O}_{\text {meas }}-\vec{O}_{\text {pred }}\right)^{T} \operatorname{cov}^{-1}\left(\vec{O}_{\text {meas }}-\vec{O}_{\text {pred }}\right)+\chi_{\text {limits }}^{2}
$$

where $\vec{O}_{\text {meas }}\left(\vec{O}_{\text {pred }}\right)$ are the measurements (predictions), cov is the covariance matrix and $\chi_{\text {limits }}^{2}$ includes the contributions from the LUX limit, the direct search at the LHC and the Higgs limits. A total of roughly $10^{9}$ different points have been scanned by the MCMC; values above 10 $\mathrm{TeV}$ for the two mass parameters and the absolute value of $A_{0}$ are not considered in this analysis. The point with the lowest $\chi^{2}=\chi_{\min }^{2}$ is identified as the global minimum and the approximate one(two)-dimensional $68 \%(95 \%)$-CL regions around that minimum are determined by requiring $\Delta \chi^{2}=\chi^{2}-\chi_{\min }^{2} \leq 1(6)$.

The expected distribution of $\chi_{\min }^{2}$ under the assumption of the cMSSM at its global minimum is determined by pseudo experiments. All measurements are smeared according to the predictions at the minimum. As re-running the whole fit with the smeared measurements is computationally impossible, the pseudo best fit point is determined from the points included in the original MCMC and the pseudo minimum $\chi^{2}=\chi_{\min , \mathrm{i}}^{2}$ is saved. This procedure is repeated roughly 1000 times in order to get a reasonably smooth distribution for $\chi_{\min }^{2}$. The $\mathrm{p}$-value is then defined as the fraction of pseudo fits with $\chi_{\min , \mathrm{i}}^{2} \leq \chi_{\min }^{2}$.

\section{Fit Results and the p-Value of the cMSSM}

With our observable set, we find a global minimum with $\chi_{\min }^{2}=30.4$ with 22 degrees of freedom. As shown in figure 1(a), the global minimum lies in a region with relatively small values of $M_{0}$ and $M_{1 / 2}$, but a second local minimum with a $\chi^{2}$ of 30.8 is observed for large values of $M_{0}$. At the global best fit point, the squark and gluino masses are close to the limits set by the LHC experiments, while due to the appearance of the focus point region in the $1 \sigma$ environment, very 
large slepton, squark and gluino masses are now also part of the favoured mass spectrum.

We obtain a p-value of $4.9 \pm 0.7 \%$ for the cMSSM at the global best fit point, which is significantly

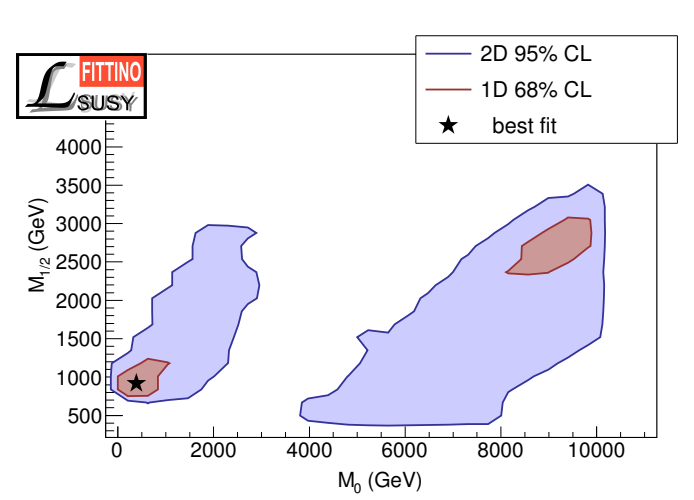

(a) The preferred parameter region in the $M_{0}-M_{1 / 2^{-}}$ plane.

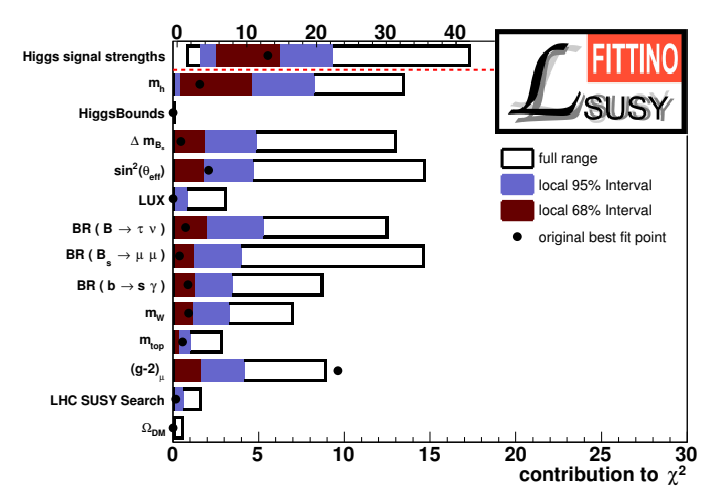

(c) Individual contributions to the minimum $\chi^{2}$ at the pseudo best fit points around the global minimum.

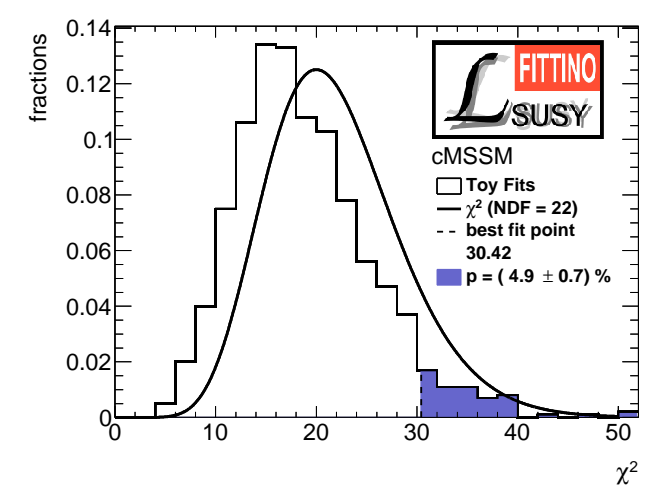

(b) Distribution of the minimum $\chi^{2}$ for the used observable set at the global minimum.

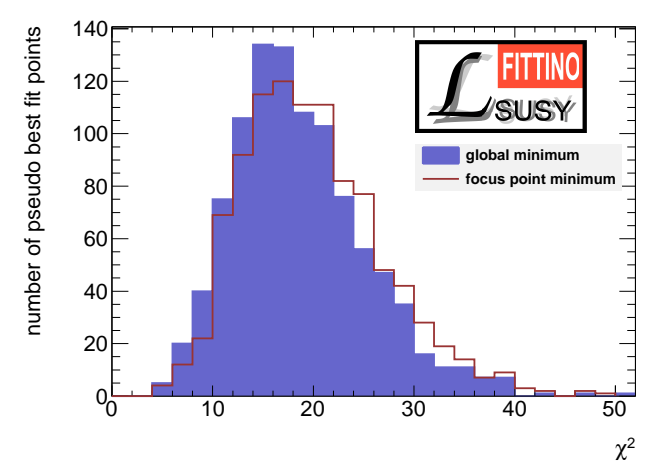

(d) Comarison of the $\chi^{2}$ distributions around the local and global minimum.

Figure 1: Fit results.

different from the value of roughly $11 \%$ that one would expect from a Gaussian $\chi^{2}$ distribution for 22 degrees of freedom, as shown in figure 1(b). To illustrate the cause for this difference, figure 1(c) shows the individual contributions of all observables to $\chi_{\min , i}^{2}$. Some contributions, like the one from HIGGSBOUNDS or the direct search at the LHC barely contribute to $\chi_{\min , \mathrm{i}}^{2}$ for any of the pseudo best fit points. This can be interpreted as an effective reduction of the number of degrees of freedom, which, compared to the Gaussian expectation, leads to a narrower $\chi^{2}$-distribution centered around smaller values.

The figure also illustrates that the smallness of the p-value is mainly driven by $(g-2)_{\mu}$, as in none of the pseudo datasets the measured value deviates as much from the prediction at the pseudo best fit point as it is the case for the real dataset.

Interestingly we find a higher p-value for the local minimum in the focus point region of $7.8 \pm 0.8 \%$. This is explained by a higher number of effective degrees of freedom in the focus point region, which causes a broader $\chi^{2}$ distribution, as shown in figure 1(d). It is mainly the direct search at the LHC that contributes, on average, with higher values to the pseudo minimum $\chi^{2}$. 


\section{Summary}

With an accurate determination of the underlying $\chi^{2}$-distribution using pseudo experiments, we exclude the cMSSM at the $90 \%$ CL. The non-observation of any SUSY like signal during the LHC Run1 along with the measurements of the Higgs boson properties pushes the preferred model parameters to values in which the cMSSM shows a similar level of disagreement between measurement and prediction of $(g-2)_{\mu}$ as the SM. It is hence the combination of very different measurements that finally allows for the global exclusion of the cMSSM - none of the observables can accomplish this alone. We also find that the obtained $\chi^{2}$-distribution differs significantly from the Gaussian expectation for the given number of degrees of freedom and, in addition, shows a significant dependence on the point around which pseudo experiments are generated. Both phenomena are related, as the effective number of degrees of freedom is different a) from the naive estimate of this number $n_{\text {constraints }}-n_{\text {parameters }}$ and $\mathrm{b}$ ) at different points in the parameter space. These results underline the necessity of the use of pseudo experiments for an accurate determination of the p-value for a model with a complex parameter space like the cMSSM when a highly non-Gaussian observable set is used. The presented results are described in more detail in [27].

\section{References}

[1] J. Wess, B. Zumino, Supergauge Transformations in Four-Dimensions, Nucl.Phys. B70, 39 (1974)

[2] H.P. Nilles, Supersymmetry, Supergravity and Particle Physics, Phys.Rept. 110, 1 (1984)

[3] P. Bechtle, T. Bringmann, K. Desch, H. Dreiner, M. Hamer, et al., Constrained Supersymmetry after two years of LHC data: a global view with Fittino, JHEP 1206, 098 (2012) [arXiv:1204.4199[hep-ph]]

[4] D. Akerib, et al., First results from the LUX dark matter experiment at the Sanford Underground Research Facility, Phys.Rev.Lett. 112, 091303 (2014) [arXiv: 1310.8214 [astro-ph] ]

[5] ALEPH, DELPHI, L3 and OPAL collaborations, Combined LEP Chargino Results, up to 208 GeV for large m0, 2001, http://lepsusy.web.cern.ch/lepsusy/www/inos_moriond01/charginos_pub.html

[6] G. Aad, et al., Search for squarks and gluinos with the ATLAS detector in final states with jets and missing transverse momentum using $\sqrt{s}=8 \mathrm{TeV}$ proton-proton collision data, JHEP 1409, 176 (2014), [arXiv:1405.7875[hep-ex]]

[7] P. Bechtle, O. Brein, S. Heinemeyer, O. Stål, T. Stefaniak, et al., HiggsBounds - 4: Improved Tests of Extended Higgs Sectors against Exclusion Bounds from LEP, the Tevatron and the LHC, Eur.Phys.J. C74(3), 2693 (2014), [arXiv:1311.0055 [hep-ph] ]

[8] P. Bechtle, S. Heinemeyer, O. Stål, T. Stefaniak, G. Weiglein, HiggsSignals: Confronting arbitrary Higgs sectors with measurements at the Tevatron and the LHC, Eur.Phys.J. C74(2), 2711 (2014), [arXiv:1305.1933[hep-ph]]

[9] W. Porod, SPheno, a program for calculating supersymmetric spectra, SUSY particle decays and SUSY particle production at e+ e-colliders, Comput.Phys.Commun. 153, 275 (2003), [arXiv: hep-ph/0301101]; W. Porod, F. Staub, SPheno 3.1: Extensions including flavour, CP-phases and models beyond the MSSM, Comput.Phys.Commun. 183, 2458 (2012), [arXiv:1104.1573 [hep-ph]] 
[10] S. Heinemeyer, W. Hollik, G. Weiglein, FeynHiggs: A Program for the calculation of the masses of the neutral CP even Higgs bosons in the MSSM,Comput.Phys.Commun. 124, 76 (2000), [arXiv:hep-ph/9812320]

[11] F. Mahmoudi, SuperIso v2.3: A Program for calculating flavor physics observables in Supersymmetry, Comput.Phys.Commun. 180, 1579 (2009), [arXiv: 0808 . 3144 [hep-ph] ]

[12] G. Belanger, F. Boudjema, A. Pukhov, A. Semenov, MicrOMEGAs: A Program for calculating the relic density in the MSSM, Comput.Phys.Commun. 149, 103 (2002), [arXiv: hep-ph/0112278]; G. Belanger, F. Boudjema, A. Pukhov, A. Semenov, micrOMEGAs: Version 1.3, Comput.Phys.Commun. 174, 577 (2006), [arXiv: hep-ph / 0405253 ]

[13] P. Gondolo, J. Edsjo, P. Ullio, L. Bergstrom, M. Schelke, et al., DarkSUSY: Computing supersymmetric dark matter properties numerically, JCAP 0407, 008 (2004), [arXiv:astro-ph/0406204]

[14] N. Nguyen, D. Horns, T. Bringmann, AstroFit: An Interface Program for Exploring Complementarity in Dark Matter Research, 2012, [arXiv:1202.1385 [astro-ph] ]

[15] M. Bahr, S. Gieseke, M. Gigg, D. Grellscheid, K. Hamilton, et al., Herwig++ Physics and Manual, Eur.Phys.J.C58, 639 (2008), [arXiv: 0803.0883 [hep-ph ] ]

[16] S. Ovyn, X. Rouby, V. Lemaitre, DELPHES, a framework for fast simulation of a generic collider experiment, 2009, [arXiv:0903.2225 [hep-ph]

[17] W. Beenakker, R. Hopker, M. Spira, PROSPINO: A Program for the production of supersymmetric particles in next-to-leading order QCD, 1996, [arXiv: hep-ph/9611232]

[18] G. Bennett, et al., Final Report of the Muon E821 Anomalous Magnetic Moment Measurement at BNL, Phys.Rev. D73, 072003 (2006), [arXiv: hep-ex/ 0602035 ]

[19] M. Davier, A. Hoecker, B. Malaescu, Z. Zhang, Reevaluation of the Hadronic Contributions to the Muon g-2 and to alpha(MZ), Eur.Phys.J. C71, 1515 (2011), [arXiv: 1010 . 4180 [hep-ph] ]

[20] S. Schael, et al., ALEPH Collaboration, DELPHI Collaboration, L3 Collaboration, OPAL Collaboration, SLD Collaboration, LEP Electroweak Working Group, SLD Electroweak Group, SLD Heavy Flavour Group, Phys.Rept. 427, 257 (2006), [arXiv: hep-ex/ 0509008 ]

[21] ATLAS Collaboration, CDF Collaboration, CMS Collaboration, D0 Collaboration, First combination of Tevatron and LHC measurements of the top-quark mass, 2014 [arXiv: 1403.4427 [hep-ex]]

[22] Tevatron Electroweak Working Group, 2012 Update of the Combination of CDF and DO Results for the Mass of the W Boson, 2012, [arXiv:1204.0042 [hep-ex] ]

[23] J. Beringer, et al., Review of Particle Physics, Phys.Rev. D86, 010001 (2012)

[24] CMS and LHCb Collaborations, Combination of results on the rare decays $B_{(s)}^{0} \rightarrow \mu^{+} \mu^{-}$from the CMS and LHCb experiments, 2013, CMS-PAS-BPH-13-007, LHCb-CONF-2013-012, CERN-LHCb-CONF-2013-012

[25] Y. Amhis, et al., Averages of B-Hadron, C-Hadron, and tau-lepton properties as of early 2012, 2012, [arXiv:1207.1158[hep-ex]]

[26] P.A.R. Ade, et al., Planck 2013 results. XVI. Cosmological parameters, Astron. Astrophys. 571, A16 (2014), [arXiv:1303.5076 [astro-ph]]

[27] P. Bechtle et.al., Killing the cMSSM softly, [arxiv:1508.05951 [hep-ph]] 